\title{
X-Ray Reflectivity from the Surface of a Liquid Crystal: Surface Structure and Absolute Value of Critical Fluctuations
}

\section{Citation}

Pershan, Peter S., and J. Als-Nielsen. 1984. X-ray reflectivity from the surface of a liquid crystal: Surface structure and absolute value of critical fluctuations. Physical Review Letters 52(9):

759-762.

\section{Published Version}

doi:10.1103/PhysRevLett.52.759

\section{Permanent link}

http://nrs.harvard.edu/urn-3:HUL.InstRepos:10360324

\section{Terms of Use}

This article was downloaded from Harvard University's DASH repository, and is made available under the terms and conditions applicable to Other Posted Material, as set forth at http:// nrs.harvard.edu/urn-3:HUL.InstRepos:dash.current.terms-of-use\#LAA

\section{Share Your Story}

The Harvard community has made this article openly available.

Please share how this access benefits you. Submit a story.

\section{Accessibility}




\title{
X-Ray Reflectivity from the Surface of a Liquid Crystal: Surface Structure and Absolute Value of Critical Fluctuations
}

\author{
P. S. Pershan \\ Gordon McKay Laboratory, Harvard University, Cambridge, Massachusetts 02138 \\ and \\ J. Als-Nielsen \\ Risd National Laboratory, DK-4000 Roskilde, Denmark \\ (Received 1 November 1983)
}

\begin{abstract}
$\mathrm{X}$-ray reflectivity from the surface of a nematic liquid crystal is interpreted as the coherent superposition of Fresnel reflection from the surface and Bragg reflection from smectic order induced by the surface. Angular dependence of the Fresnel effect yields information on surface structure. Measurement of the intensity of diffuse critical scattering relative to the Fresnel reflection yields the absolute value of the critical part of the density-density correlation function.
\end{abstract}

PACS numbers: $61.30 .-\mathrm{v}, 61.10 . \mathrm{Fr}$

Although there has been considerable recent interest in both structure and critical phenomena at all types of interfaces there have been very few experimental techniques available for probing the microscopic properties of liquid-vapor interfaces. ${ }^{1-10}$ One purpose of this manuscript is to demonstrate that, with use of a synchrotron source, $x$-ray reflectivity from liquid surfaces is a practical technique for obtaining structural information at the molecular level. In the particular case of the nematic liquid crystal octyloxycyanobiphenyl (8OCB) using $\lambda=1.54 \AA$, the observed reflection follows the Fresnel reflection formulas ${ }^{11,12}$ for incident angles between grazing and $1.3^{\circ}$, independent of temperature. For larger angles surface structure and Bragg reflection from surface-induced smectic order (i.e., layering) result in temperature-dependent deviations.

A second purpose of the manuscript is to demonstrate that since the $\mathrm{x}$-ray reflectivity can be calculated from the Fresnel formulas, ${ }^{11}$ the ratio of the scattering from critical fluctuations in the nematic phase of bulk $80 C B$ to the signal reflected from the surface yields the absolute value of the critical part of the density-density correlation function. Similarly the absolute value of the surface-induced smectic order in the nematic phase is also measured.

The experiments have been carried out at HASYLAB in Hamburg, Germany, and details of the spectrometer have been reported previous$1 y 0^{12,13}$ The geometry is shown schematically in Fig. 1. The horizontal synchrotron beam is deflected downward by an angle $\theta$, with a spread in the vertical direction $\Delta \theta_{1}$, to produce the incident wave vector $\vec{k}_{1}$ 。 The height of the horizontal liquid crystal surface is controlled such that the beam strikes a fixed position on the sample. The detector assembly can be either a tilted crystal or a simple slit arrangement. In either case, if the spectrometer is tuned for specular reflection the wave vector of the detected beam, $\overrightarrow{\mathrm{k}}_{2}$, is inclined upward by the same average angle $\theta$. The detector accepts an angular spread $\Delta \theta_{2}$ in the vertical direction and $\Delta \psi_{2}$ in the horizontal out-of-plane direction. If the detector is centered on the plane of the figure the average scattering vector $\overrightarrow{\mathrm{Q}}=\overrightarrow{\mathrm{k}}_{2}-\overrightarrow{\mathrm{k}}_{1}=\hat{z} 2 \mathrm{k} \sin \theta$. Moving the detector out of the plane scans $Q_{x}$. The spectrometer records all scattered $x$ rays for which $\vec{Q}$ is contained in the resolution volume $\Delta^{3} Q$ indicated by the parallelogram.

As discussed previously, ${ }^{13}$ a consequence of
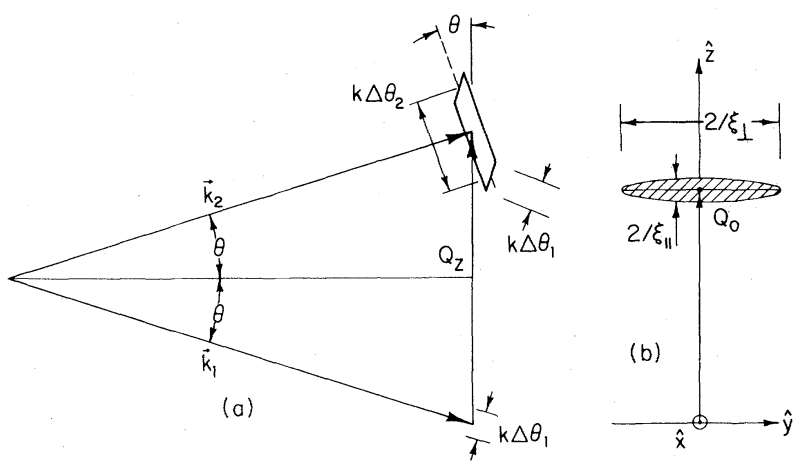

FIG. 1. (a) Schematic of the spectrometer geometry. Typically $\lambda=1.54 \AA, \theta \simeq 1.5^{\circ}, \Delta \theta_{1} \simeq 0.003^{\circ}$, and $\Delta \theta_{2}$ $\approx 0.01^{\circ}$ and $0.73^{\circ}$ for high and low resolution, respectively. The out-of-plane angular resolution $\Delta \psi_{2}$ is typically $0.003^{\circ}$ and $0.4^{\circ}$ for high and low resolution, respectively. (b) Schematic shape of the full width at half maximum for critical scattering from the bulk nematic phase. 


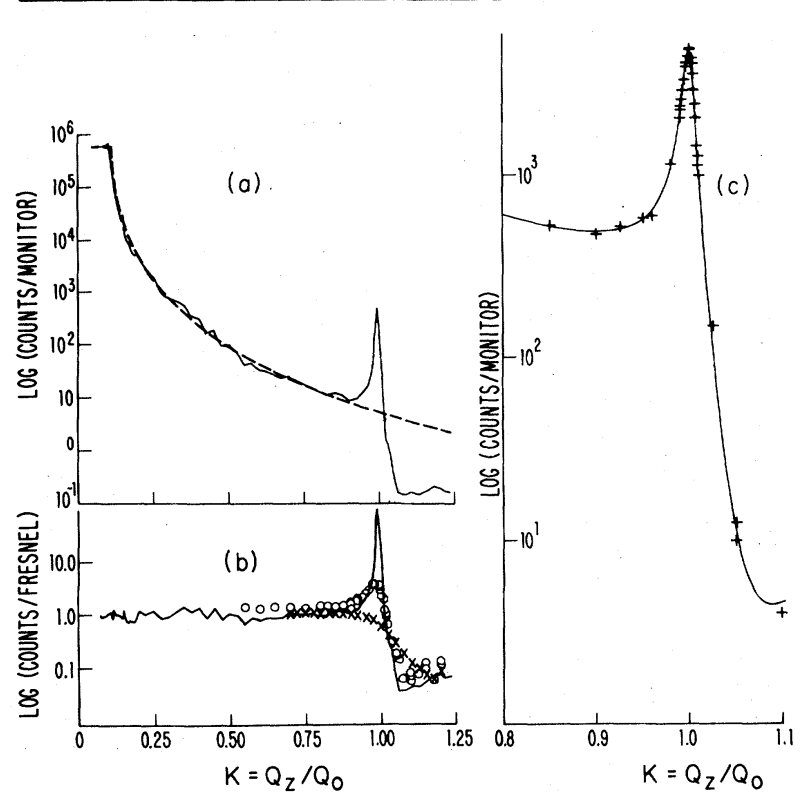

FIG. 2. (a) High-resolution scan of the reflected intensity from the surface of $80 \mathrm{CB} ; T-T_{N A}=0.05^{\circ} \mathrm{C}$. The peak at $K=1$ corresponds to $Q_{z}=Q_{0}=0.1989 \AA^{-1}$. The dashed line is the calculated Fresnel reflection law. (b) Reflected intensity vs $K_{z}$ at high resolution divided by the Fresnel law. The solid line is for $T$ - $T_{N A}=0.05^{\circ} \mathrm{C}$; open circles and crosses are for $T$ $-T_{N A}=2.8$ and $11.06^{\circ} \mathrm{C}$, respectively. (c) High-resolution scan, $T-T_{N A}=0.40^{\circ} \mathrm{C}$. The solid line is the fit by the model.

translational invariance parallel to the surface, i.e., in the $x-y$ plane, is that the scattering cross section for $x$ rays that are either specularly reflected from the flat surface, or Bragg reflected from smectic layers parallel to it, is proportional to $\delta\left(Q_{x}\right) \delta\left(Q_{y}\right)$. A spectrometer with small $\Delta^{3} Q$ discriminates against diffuse scattering from the bulk liquid crystal. The solid line in Fig. 2(a) displays a high-resolution scan from $K=Q_{z} / Q_{0}$ $\simeq 0.07$ to $\simeq 1.25$ at $T-T_{N A}=0.050{ }^{\circ} \mathrm{C}$. The dashed line indicates the calculated Fresnel law for radiation incident on a planar dielectric discontinuity. Figure 2(b) displays the result of dividing the data by the Fresnel reflection law $R_{\mathrm{F}}(K)$ for temperatures $T-T_{N A}=0.05,2.8$, and $11.06{ }^{\circ} \mathrm{C}$. The peak at $K=Q_{z} / Q_{0}=1$ is due to smectic layers localized to a depth of $\xi_{\|}(T)$ below the surface. ${ }^{13}$ The full width at half maximum $\Delta Q_{z} / Q_{0}$ is equal to $2 / Q_{0} \xi_{\|}(T)$, where $\xi_{\|}(T)$ is the longitudinal correlation length of the bulk nematic ${ }^{14}$; see Fig. 1(b). For $K \lesssim 0.9$ the reflected intensity is essentially described by the Fresnel law independent of temperature. For $K \gtrsim 1.05$ it falls below the Fresnel value. For the scan displayed in
Fig. 2(c), $T-T_{N A}=0.40^{\circ} \mathrm{C}$, the condition $Q_{x}=Q_{y}$ $=0$ was continuously monitored for each $Q_{z}$ and scattering that did not obey the selection rule was subtracted. The solid line in Fig. 2(c) is the result of fitting the data by the convolution of the experimental resolution with the cross section of the model to be described below.

Standard treatments for the reflection coefficient from a surface with structure yield $R\left(Q_{z}\right)$ $=R_{\mathrm{F}}\left(Q_{z}\right)\left|\Phi\left(Q_{z}\right)\right|^{2}$, where $\Phi\left(Q_{z}\right)=(1 / \bar{\rho}) \int_{-\infty}^{\infty}(d \rho / d z)$ $\times \exp \left[-i\left(Q_{z} z\right)\right] d z .^{15,16}$ The density is taken as the sum of two terms, $\rho(z)=\rho_{0}(z)+\rho_{1}(z)$. The sharp peak at $K \simeq 1$ is due to smectic order induced by the surface ${ }^{13} ;(\bar{\rho})^{-1} d \rho_{0} / d z=B_{s} Q_{0} \sin \left(Q_{0} z\right) \exp (-z /$ $\left.\xi_{\| 1}\right)^{13}$ gives a Lorentzian-type term, $\Phi_{0} \simeq+\left(B_{s} Q_{0} /\right.$ 2) $\left\{+\left(Q_{z}+Q_{0}\right)^{-1}-\xi_{\|}\left[\left(Q_{z}-Q_{0}\right) \xi_{\|}-i\right]^{-1}\right\}$. Data of the type shown in Fig. 2(b) demand that the Fourier transform of the second term be flat for $K \lesssim 0.8$ and fall off reasonably rapidly in the vicinity of $K \simeq 1$. One form that has this property is $\Phi_{1}\left(Q_{z}\right)$ $=0.5\left\{\operatorname{erf}\left[\left(Q_{z}+Q_{1}\right) / \sqrt{2} \sigma\right]-\operatorname{erf}\left[\left(Q_{z}-Q_{1}\right) / \sqrt{2} \sigma\right]\right\}$, with $Q_{1} / Q_{0} \approx 1$ and $\sigma / Q_{0} \ll 1$. The Fourier transform of this is $(\bar{\rho})^{-1} d \rho_{1} / d z=(\pi z)^{-1} \exp \left[-0.5(\sigma z)^{2}\right]$ $\times \sin Q_{1} z$. It describes a surface of width $\sim \pi / Q_{1}$, followed by oscillations that decay as $z$ increases.

The nonlinear least-squares fit shown in Fig. 2 (c) yields $Q_{1} / Q_{0}=1.04 \pm 0.01, \sigma / Q_{0}=0.062 \pm 0.015$, $B_{s}=0.067 \pm 0.003$, and $\xi_{\|} Q_{0}=145 \pm 3$ with a $\chi^{2}=6$. The value of $\xi_{\|} Q_{0}$ agrees with published values for $80 \mathrm{OB} .{ }^{13,14}$ There is a previous measurement of the temperature dependence of $B_{s}$; however, this is the first measure of its absolute value. ${ }^{13}$ Although the specific function chosen for $\Phi_{1}$ is $a d$ hoc the general form dictated by the data results in a $\rho_{1}(z)$ with smectic density oscillations near the surface. The sum of $\rho_{0}+\rho_{1}$ thus gives a nonexponential decay of the smectic order. Since $B_{s} \xi_{\|} Q_{0}$ diverges as $T-T_{N A}$, the peak intensity is essentially given by $\left|\Phi_{0}\right|^{2}$, and accurate knowledge of $\Phi_{1}$ is not necessary for determination of the critical properties of $\boldsymbol{B}_{s}$. Asymmetry between the intensities in the wings for $K>1$ and $K<1$ is due to interference effects between $\Phi_{0}$ and $\Phi_{1}$.

A second method of taking data, in which spectrometer alignment is less critical, is to lower the resolution by increasing the height and width of the detector slit. In Fig. 1 this means increasing $\Delta \theta_{2}$ and also the slit width along the $\hat{x}$ direction. As the resolution volume is scanned in the vertical direction it sweeps out a strip in reciprocal space of width $\theta \times\left(\Delta \theta_{1}+\Delta \theta_{2}\right) k$ thereby integrating over a typical value of $\Delta Q_{y} \cong 0.003 Q_{0}$. In addition to integrating over $Q_{x}$ the wider slit increases the intensity of diffuse critical scatter- 
ing relative to the sharp surface scattering. With neglect of $Q_{\perp}{ }^{4}$ corrections, the cross section for diffuse critical scattering is proportional to $S_{0}(\vec{Q})$ $=\sigma_{0} /\left[1+\xi_{\|}\left(Q_{z}-Q_{0}\right)^{2}+\xi_{\perp}{ }^{2} Q_{\perp}{ }^{2}\right]$, where $S_{0}(\vec{Q})$ is defined to have the units of volume. The ratio of critical scattering to Fresnel reflection is obtained by integrating

$$
I(\vec{Q})=\left(Q^{4} \beta / 32 \pi^{2}\right) S_{0}(\vec{Q}) /\left(Q k^{2} \cos \theta \Delta \theta_{1}\right)
$$

over the resolution volume $\Delta^{3} Q$ defined by the slits, where $\beta=0.2 \mathrm{~cm}$ is the absorption length in $80 \mathrm{CB}$ for $8 \mathrm{-keV} x$ rays. In general, $\xi_{\|} / \xi_{\perp} \sim 10$ and the half-intensity contour of $I(\vec{Q})$ has the qualitative form sketched in Fig. 1(b). As $Q_{z}-Q_{0}$ is scanned through zero with $2 / \xi_{\perp} \gg \Delta Q_{y}$, there is a range for which the convolution of the resolution volume and $I(\vec{Q})$ is approximately given by $\iint_{-\infty}^{\infty} I(\vec{Q})$ $\times d Q_{x} d Q_{z}$, essentially independent of $2 \theta_{0}$. The spectral shape of the signal from the bulk has a flat top, falling off sharply for $\left|Q_{z}-Q_{0}\right| \gtrsim \Delta \theta_{\mathbf{2}} k / 2$. Figure 3(a) displays data observed with the lowresolution geometry for three temperatures. The solid line is a theoretical curve that results from the sum of the surface signal $|\Phi(Q)|^{2}$ plus the result of integrating Eq. (1) over the measured resolution volume. This interpretation is confirmed by comparison of scans in which a narrow detector slit $\left(\Delta \psi_{2} \simeq 0.07^{\circ}\right)$ is moved out of the scattering plane, i.e., in the $Q_{x}$ direction. Figure $3(\mathrm{~b})$ displays a transverse scan at $T-T_{N A}=0.796{ }^{\circ} \mathrm{C}$, with $K_{z}=1.0$. The narrow peak at $Q_{x}=0$ is due to the surface and the broader peak to the bulk. The solid line in Fig. 3(b) is obtained with use of previously measured values of $\xi_{\|}(T)$ and $\xi_{\perp}(T)^{14}$ and integration of $S_{0}(\vec{Q})$ (including $Q_{\perp}{ }^{4}$ correc-
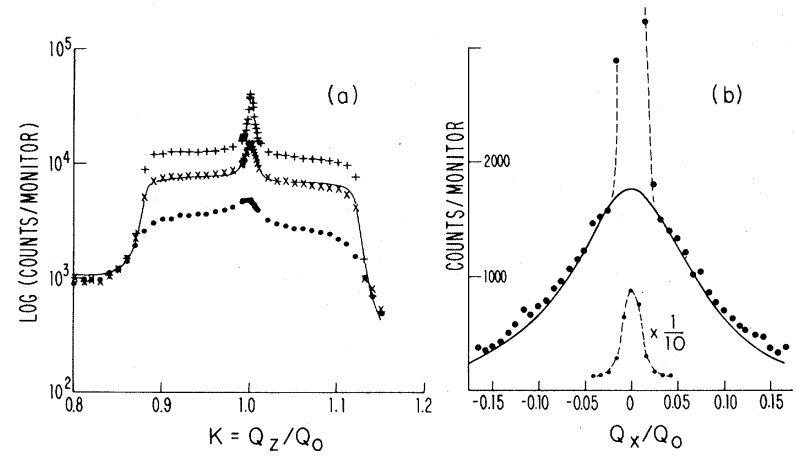

FIG. 3. (a) Low-resolution signals vs $K$ for $T-T_{N A}$ $=0.011,0.164$, and $1.105^{\circ} \mathrm{C}$, represented by plusses, crosses, and circles, respectively. The solid line through the $0.164^{\circ} \mathrm{C}$ data is the theoretical model discussed in the text. (b) Transverse out-of-plane scans for the same height as in (a) but with narrow width, $\Delta \psi_{2}=0.07^{\circ}$. tions) over the resolution volume.

From the data in Fig. 3(a) and other similar data, nonlinear least-squares fits with only two adjustable parameters yield $B_{s} \xi_{\|} Q_{0}=(0.45$ $\pm 0.02) t^{-(0.43 \pm 0.04)}$ and $Q_{0}{ }^{3} \sigma_{0}=(0.039 \pm 0.002)$ $\times t^{-1.26 \pm 0.01} ; t=\left(T / T_{N A}\right)-1{ }^{17}$ The temperature dependence of $\sigma_{0}$ is consistent with previous measurements of bulk $8 \mathrm{OCB}^{14}$; however, the absolute value of $\sigma_{0}$ has not previously been measured.

The physical significance of $\sigma_{0}$ is better appreciated upon taking the Fourier transform of $S(Q)$. Defining $R^{2}=\left[x^{2}+y^{2}+\left(\xi_{\perp} z / \xi_{\|}\right)^{2}\right]\left(Q_{0} / 2 \pi\right)^{2}$ one obtains $(\bar{\rho})^{-2}\langle\delta \rho(\overrightarrow{\mathrm{r}}) \delta \rho\rangle \simeq\left[\sigma_{0} Q_{0}{ }^{3} / 4 \pi^{2}\left(Q_{0} \xi_{\|}\right)\left(Q_{0} \xi_{\perp}\right)\right] R^{-1}$ $\times \exp \left(-2 \pi R / Q_{0} \xi_{1}\right) \cos \left(Q_{0} z\right)$. Since $\xi_{\|} \sim t^{-0.71 \pm 0.04}$ and $\xi_{\perp} \sim t^{-0.58 \pm 0.04}$, the ratio $\sigma_{0} / \xi_{\|} \xi_{\perp} \sim t^{-0.03 \pm 0.06}$ is essentially temperature independent. ${ }^{14}$ The same is true for the five other materials for which published data exist. ${ }^{18}$ Thus the absolute value of $\sigma_{0} Q_{0} / 4 \pi^{2} \xi_{\|} \xi_{\perp}=(0.26 \pm 0.01) \times 10^{-2} t^{0.03 \pm 0.06}$ is a useful measure of the absolute value of the critical part of the density-density correlation function.

To summarize, we have demonstrated the utility of $\mathrm{x}$-ray reflectivity to obtain information on fluid surfaces. In particular, the $Q_{x}=Q_{y}=0$ selection rule facilitates empirical separation of surface reflectivity from other scattering processes. Further, we have demonstrated that since the Fresnel reflection laws can be applied to $x$-ray wavelengths, the absolute intensity of other scattering processes can be obtained from their intensity relative to Fresnel reflection. For the liquid crystal $8 \mathrm{OCB}$ we have demonstrated that the interference between density oscillations localized at the surface and surface-induced smectic order that decays into the bulk as $\exp (-z)$ $\left.\xi_{\|}\right)$is responsible for the shape of the peak at $K$ $\simeq 1$. It is tempting to try to explain the contraintuitive temperature dependence of $B_{s}{ }^{13}$ with Landau-type mean-field theory containing a term $\delta F \simeq a(t) \rho_{1}$ capable of inducing coherent smectic order. A naive guess might take $a \sim t$ since the surface would quench phase fluctuations that are responsible for renormalization effects in the bulk. One might then expect $\rho_{0} \sim\left(t \xi_{\|}\right) \exp \left(-z / \xi_{\|}\right)$ $\times \cos \left(Q_{0} z\right)$ or $B_{s} \xi_{\|} Q_{0} \simeq t^{-0.42 \pm 0.06}$. This is in fact, the observed temperature dependence. Finally, we have obtained a value for the critical part of $\langle\delta \rho(r) \delta \rho\rangle$.

The excellent research conditions provided by HASYLAB and the competent assistance of Ris $\varnothing$ technical staff E. Dahl Petersen, S。 Bang, J. Linderholm, and J. Munck are gratefully acknowledged. Alan Braslau, Kelby Chan, and Alex Weiss assisted in some of the measurements and 
data analysis. This work was supported in part by grants from the Danish National Science Foundation, by the Ris $\varnothing$ National Laboratory, by the National Science Foundation through Grant No. DMR 82-12189, and by the Joint Services Electronics Program (U。 S. Army, Navy, and Air Force) through Grant No. N00014-75-C-0648.

${ }^{1}$ E. Brezin, B. I. Halperin, and S. Leibler, Phys. Rev. Lett. 50, 1387 (1983).

${ }^{2} \mathrm{~J}$. Rudnick and D. Jasnow, Phys. Rev. Lett. $\underline{48,} 1059$ (1982).

${ }^{3}$ M. Robert and C. Stuart, Phys. Rev. Lett. 49,1434 (1982).

${ }^{4}$ S. Alvarado, M. Campagna, and H. Hopster, Phys. Rev. Lett. 48, 51 (1982).

${ }^{5}$ R. Lipowsky, Phys. Rev. Lett. 49 , 1575 (1982).

${ }^{6}$ H. Nakanishi and M. E. Fisher, Phys. Rev. Lett. $\underline{49}$, 1565 (1982).

${ }^{7}$ C. Frank and S. E. Schnatterly, Phys. Rev. Lett. $\underline{48}$, 763 (1982).

${ }^{8}$ D. Beysens and S. Leibler, J. Phys. (Paris), Lett. 43, L133 (1982).

${ }^{9}$ M. P. D'Evelyn and S. A. Rice, Phys. Rev. Lett. 47,
1844 (1981); D. S. Sluis, M. P. D'Evelyn, and S. A. Rice, J. Chem. Phys. 78, 1611 (1983).

${ }^{10} \mathrm{~J}$. Meunier and D. Langevin, J. Phys. (Paris), Lett. 43 , L185 (1982).

${ }^{11}$ See, for example, G. H. Vineyard, Phys. Rev. B $\underline{26}$, 4146 (1982), or D. Oxtoby, F. A. Novak, and S. A.

Rice, J. Chem. Phys. 76, 5278 (1982).

${ }^{12} \mathrm{~J}$. Als-Nielsen and P.S. Pershan, Nucl. Instrum. Methods 208, 545 (1983).

${ }^{13} \mathrm{~J}$. Als-Nielsen, F. Christensen, and P. S. Pershan, Phys. Rev. Lett. 48, 1107 (1982).

${ }^{14}$ J. D. Litster, J. Als-Nielsen, R. J. Birgeneau, S. S. Dana, D. Daridov, F. Garcia-Golding, M. Kaplan, C. R. Safinya, and R. Schaetzing, J. Phys. (Paris), Colloq. 40, C3-339 (1979).

${ }^{15} \mathrm{P}$. Beckmann and A. Spizzichino, The Scattering of Electromagnetic Waves From Rough Surfaces (MacMillan, New York, 1963).

${ }^{16}$ E. S. Wu and W. W. Webb, Phys. Rev. A $\underline{8}, 2065$ (1973).

${ }^{17}$ Quoted error estimates do not include the systematic error due to $\pm 0.005^{\circ} \mathrm{C}$ uncertainty in what we estimate to be the variation in sample temperature normal to the surface. This is most serious for large $\xi_{\|}$as $T \rightarrow T_{N A}$.

${ }^{18} \mathrm{C}$. W. Garland, M. Meichle, B. M. Ocko, A. R. Kortan, L. J. Yu, D. Litster, and R. J. Birgeneau, Phys. Rev. A 27, 3234 (1983). 